\title{
Hacia la dinamización del currículo: necesidad de la escucha, la sospecha y la renovación ${ }^{*}$
}

\section{Towards the revival of the curriculum: the need listening, suspicion and renovation}

\author{
Juan José Burgos Acosta*
}

Las teorías curriculares son teorías sociales, no sólo porque reflejan la historia de las sociedades en las que surgen, sino también en el sentido de que están vinculadas con posiciones sobre el cambio social y, en particular, con el papel de la educación en la reproducción o transformación de la sociedad.

Kemmis

Fecha de recepción: 7 de febrero de 2011

Fecha de revisión: 22 de febrero de 2011 Fecha de aprobación: 28 de marzo de 2011

* Artículo de investigación científica, fruto del trabajo en la línea de Pedagogía adscrita al grupo Ciencia-espiritualidad, clasificado en $\mathrm{C}$ por Colciencias.

** Doctor H.C. en Filosofía de la Educación. Estudiante Doctorado en Educación de la Universidad Santo Tomás. Magíster en Educación y magíster en Estudios Políticos. Correo electrónico: chasjjba14@yahoo.es 


\title{
Resumen
}

Los procesos educativos que se generan en el interior de la escuela no pueden desconocer que están afectados por el entorno social y cultural que les exige revisar constantemente las formas como se llevan a cabo las prácticas educativas en los escenarios de la vida cotidiana. En virtud de esa constante dinámica, el currículo se convierte en una estrategia fundamental para fortalecer la educación integral y responder críticamente a los desafíos que impone el mundo de hoy, que van desde los aprendizajes cognoscitivos hasta la convivencia democrática y el impacto que producen en la sociedad.

Palabras clave: currículo, sociedad, transformación, formación integral.

\begin{abstract}
Educational processes which are generated within school cannot ignore they are affected by a social and cultural environment that requires them to constantly review the ways in which educational practices are conducted in everyday life scenarios. In virtue of this dynamic, curriculum becomes a key strategy for strengthening integral education and responding critically to the challenges posed by the world of today, ranging from cognitive learning to democratic coexistence and the impact that it produces in society.
\end{abstract}

Keywords: curriculum, society, transformation, integral formation.

\section{Introducción}

La comprensión del currículo desde una perspectiva anclada en dimensiones como la racionalidad, la afectividad y el valor del pensamiento subjetivo conduce hacia un marco de interpretación que transita por caminos distintos a los propuestos por la educación tradicional, que ha estructurado su discurso sobre las bases de racionalidad instrumental, pues al centrarse en la individualidad del sujeto y su capacidad para construir mundos posibles a partir de la interpretación de su propio presente, la acción individual y colectiva cobra nuevos sentidos para realizar acciones encaminadas a la superación de las tradicionales formas de aprender, que van más allá de un programa y se sumergen en el mundo de la vida cotidiana en donde todas las prácticas educativas tienen una intención, un sentido, y se orientan hacia una 
formación que integre todos los aspectos que conforman al ser humano y sus relaciones consigo mismo, con el mundo, con la naturaleza y con lo trascendente, espiritual. De acuerdo con estas consideraciones, este ensayo pretende, en primer lugar, ofrecer una reflexión en torno al currículo que parte de las herramientas que aporta la hermenéutica para su comprensión. En segundo lugar, esa dimensión de que la hermenéutica lleva a la consideración de una formación que sea integral, de manera que no deja ninguna dimensión por fuera de los aprendizajes. En un tercer momento se muestra el valor formativo que tienen lo racional y lo emocional, como aspectos fundamentales para la construcción del currículo. Y finalmente se ofrecen unas conclusiones orientadoras sobre los criterios generales que deben tenerse en cuenta en la concepción y práctica de un currículo para el siglo XXI.

\section{Desarrollo}

\section{Las herramientas de la hermenéutica}

Que se comprenda la educación como dirigida hacia la formación es ya una opción determinante que compromete con la permanente construcción del ser de la persona y, en consecuencia, con el proyecto de humanidad en el que se está inscrito. Implica que la razón de ser de la educación no puede ser otra que la formación humana, que su tarea consiste en impregnar los procesos educativos de valor formativo, y que su intención es elucidar las condiciones humanas de cada ser y de todos como humanidad, posibilitando la continua creación de mundos con sentido, lo cual será siempre una aventura, y a la vez un riesgo que se tendrá que correr. Aventura porque es una búsqueda constante llena de incertidumbres y retos; y riesgo de perder ciertas "seguridades" porque se trata, en ocasiones, de dejar ciertos paradigmas que mantienen anclado el pensamiento y la práctica educativa y no permiten avanzar hacia nuevos horizontes. Ese tránsito está guiado siempre por la pregunta, ya que ésta abre caminos nuevos.

... Gadamer nos enseñó que es posible educarse y educar en el espíritu libre y en la lógica de la pregunta: nos educamos en la pregunta; nos formamos en ella y nos damos cuenta que, en últimas, educar es educar en la pregunta y para la pregunta (Sopó, 2002: 122).

Decir aventura es afirmar que la educación -si en realidad se quiere plantear como una respuesta a las generaciones venideras- no es algo terminado, concluido, sino en permanente búsqueda de su propio ser. No hay patrones absolutos. Cualquiera que viva inmerso y comprometido con el espacio educativo sabrá que los retos que impone la actual sociedad demandan de cada uno de los pedagogos una enorme flexibilidad, una reserva de estrategias creativas, capaces de poner en sintonía sus aspiraciones con los deseos de sus estudiantes y de la comunidad educativa en general. Por ende, el currículo contribuye para que se puedan construir o instaurar cotidianamente mapas, pistas, taxonomías, modelos, sobre diversos aspectos pedagógicos. Algunas de esas tareas curriculares pueden evidenciarse, por ejemplo, en el diseño de otros tipos de evaluación, no necesariamente cuantitativos: 
colores, emblemas, símbolos; otras modalidades de trabajo, la creación que no sigue un modelo, la invención como ejercicio de la libertad, las asociaciones o complicidades según intereses; otras metodologías de clase, la exposición al estilo de la plástica como socialización de las producciones, las charlas como motivación para la complejidad y no como reducción a la simpleza; otras concepciones o posturas ideológicas sobre el docente, la superación del informador por el cuestionador, del educador como replicante al educador como productor o escritor de sus propuestas personales; otros ritmos de clase, la diversificación de los tiempos en el aula, la inclusión de la polifonía como práctica de la armonía desde la diferencia; otros lenguajes, el humor y la ironía como estrategias discursivas de contacto; la inclusión o el intercambio del mundo presente en las telenovelas, en la música, en la moda y en los lenguajes juveniles, con el mundo escolar; combinatorias entre la vida cotidiana y la vida académica, etc.

Al mismo tiempo, avalar la educación desde la aventura es propiciar un currículo de riesgo, de incertidumbre y complejidad; en suma, es abonar el terreno para la creatividad. Vistas así las cosas, poner a marchar el(los) currículo(s) ayuda a entender las distintas poéticas, en cuanto "hacer con sentido", a ver cuáles son las etapas de un proceso creativo, a ver sus diferencias, a descubrir sus alcances. El enfoque innovador del currículo, entonces, muestra cuáles son las herramientas didácticas de que se vale un maestro, una institución, para hacer más creíble el proceso educativo. Una vez desentrañadas esas intencionalidades internas, podrán dar luces sobre cómo emplearlas en el campo educativo. De ese modo el arte y la pedagogía se imbrican constantemente.

Según lo anterior, es posible que para este análisis sirvan como horizonte de comprensión los aportes que desde la hermenéutica ofrece Paul Ricoeur, a partir de los cuales se pueden "enganchar" las reflexiones sobre el currículo y su relación con los procesos de formación y transformación histórica. Para tal efecto, lo primero que hay que hacer es seguir de cerca sus consejos.

\begin{abstract}
Entendemos que la interpretación tiene una historia y que esa historia es un segmento de la tradición misma; siempre se interpreta desde algún lugar, para explicar, prolongar $y$, de ese modo, mantener viva la tradición misma en la cual estamos. Así, el tiempo de la interpretación pertenece, en cierta forma, al tiempo de la tradición. Pero, como contrapartida, la tradición, aún entendida como transmisión de un depositum, es tradición muerta si no se interpreta continuamente ese depósito: una herencia no es un paquete cerrado que pasa de mano en mano sin ser abierto, sino más bien un tesoro del que se extrae a manos llenas y que se renueva en la operación misma de agotarlo (2008: 31).
\end{abstract}

Lo expresado aquí lleva a combinar, al mismo tiempo, una voluntad de escucha y una voluntad de sospecha frente a los diversos escenarios educativos donde se esté trabajando. Tal recomendación conlleva una serie de tareas como la de leer o mirar muchas veces los territorios que se están interviniendo, comparar visiones sobre el mundo; en suma, manipular exhaustivamente, desde el punto de vista pedagógico, 
todas las prácticas educativas a que haya lugar, para que prontamente se estén renovando, actualizando, innovando. La voluntad de escucha, además, obliga a poner lo que Ricoeur llama la unidad cultural en perspectiva de tradición y a la vez de cambio; hay que transformar y a la vez mantener aquello que no se puede dejar de lado, porque se está convencido que esas llamadas "tradiciones" continúan fecundando la formación.

Se trata de poder ubicar, hasta donde sea posible, el momento, el contexto, los mojones históricos a través de los cuales cobra sentido el territorio de una propuesta curricular. Este esfuerzo de voluntad de escucha tiene como fin evitar las interpretaciones superficiales que denotan no sólo ignorancia sino incapacidad para situar en contexto el objeto del análisis y de las prácticas educativas. A este respecto, Portela sugiere que:

... un currículo es producto de la interiorización y mediación de lenguajes de los mundos simbólicos, para la solución de tres conflictos fundamentales: el ser, el lenguaje y el conocimiento como coordenadas a construir, a partir de procesos de cooperación, e interpretación (2003: 4).

El otro punto, que para el análisis sobre las relaciones entre currículo, sociedad y cultura orienta de manera importante esta disertación, mencionado por Ricoeur, corresponde a la voluntad de sospecha. Ahora se trata de someter lo que él llama "la unidad cultural" a la paciente y fina mirada escrutadora de la duda. Trabajo con las ideologías, con las mentalidades, con los imaginarios. Se trata de desenmascarar el sentido; de pasar de la superficie al nivel profundo tal unidad. De hacer una especie de genealogía o arqueología. Si en un primer momento se escuchan las voces en su contexto, ahora se trata de oírlas a la luz de los tiempos de hoy. La voluntad de sospecha, entonces, necesita construir un dispositivo, un modelo conceptual capaz de hacer comprensibles los elementos dispersos de lo que se debate. Sospechar es tanto como necesitar de un método, de un andamiaje de pensamiento capaz de hacer emerger lo oculto o sepultado. Lo importante es que la voluntad de sospecha necesita, para ser vigorosa, organizarse, constituirse de manera sistemática, tornarse en una práctica sometida a escrutinio. Lo que implica un constante diálogo intersubjetivo para construir consensos y abandonar de plano toda intención de imponer una verdad. Es decir, es un proceso horizontal que pone en los escenarios educativos a dialogar, a discutir, a debatir sobre qué es lo más conveniente y lo mejor para una formación en los diversos contextos. Habermas lo enuncia de la siguiente manera:

Las interacciones sociales son más o menos cooperativas o estables, más o menos conflictivas o inestables. La cuestión teórico-social de cómo es posible el orden social se corresponde con la cuestión de teoría de la acción de cómo al menos dos participantes en la interacción pueden coordinar sus planes de acción de forma que alter puede "enganchar" sus acciones en las de ego sin conflictos $y$, en todo caso, evitando el peligro de una ruptura de la interacción [...]. El concepto de la acción comunicativa está pensado de tal manera que los actos del entendimiento que vinculan los planes de acción de diversos participantes y que resumen las acciones orientadas a 
un objetivo en una relación interactiva no pueden retrotraerse, por su parte, a una acción teleológica. Los procesos de entendimiento buscan un acuerdo que depende de la aprobación, racionalmente motivada, del contenido de una aceveración. No es posible imponer el acuerdo a la otra parte, ni se le puede imponer al interlocutor mediante una manipulación. El acuerdo descansa siempre sobre una convicción conjunta (2008: 138-139).

Si no hay un método, si el pensamiento crítico no está estructurado, con mucha dificultad la voluntad de sospecha superará el mero pálpito o el comentario gratuito. Sin método no hay posibilidades de descubrir el rostro oculto tras la máscara, es decir, todo lo que se esconde detrás de una propuesta curricular de formación. Pero ese proceso no lo hace una sola persona sino una comunidad donde todos sean tratados como sujetos y no como objetos del conocimiento. De ahí la necesidad de reconocer que el sujeto es lo propio de todo hombre, no sólo mío. Al lado de un sujeto como yo, existe un tú (sujeto), un él (otro sujeto) y un nosotros (otros sujetos). Y cada uno de ellos es un sujeto; es decir, otro sujeto que merece respeto, que debe ser incluido, valorado y dignificado en la cultura donde vive. Se ha de tener en cuenta que ese sujeto, que esos sujetos, también son parte de la comunidad educativa y están en posibilidad de participar en la construcción y ejecución del currículo, de formarse para vivir democráticamente y aportar significativamente a procesos de transformación histórica. Es decir, una concepción del currículo abierta, incluyente y democrática no trata al sujeto como un instrumento, sino como parte vital de un conjunto comunitario de sujetos capaces de autodeterminación. En palabras de Touraine: "resulta imposible separar al individuo de su situación social. Hay que oponer al individuo consumidor de normas y de instituciones sociales al individuo productor de esa vida social y sus cambios" (2000: 232). Es decir, la formación de la conciencia crítica ha de ser un eje transversal del currículo en la medida en que se convierte en una herramienta de vital importancia para lograr cambios significativos que sean reflejos también de la capacidad de autonomía y libertad de las comunidades educativas. En esa línea, Giroux dice:

\begin{abstract}
En la educación necesitamos espacios para la expresión, para la libertad, un espacio público en el que las personas vivientes puedan reunirse para hablar críticamente y actuar, cada una libre de articular una perspectiva distintiva, y todas las cuales se le conceda el mismo valor. Debe ser un espacio de diálogo, un espacio donde se pueda tejer una red de relaciones y donde se pueda crear un mundo común que continuamente se renueve (2006: 160).
\end{abstract}

Se trata, pues, de hacer una especie de reconstrucción, de rearmar lo que está aparentemente "marchando bien" para reorientarlo, para darle vida, para dinamizarlo y de esa manera aportar a la transformación en la educación y, por ende, a la superación de aquellos conflictos que pueden desvanecer el sentido de la formación integral. Magendzo dice, a propósito de lo anterior:

La concepción de reconstrucción social, como su nombre lo indica, plantea la necesidad de hacer del currículo una instancia para reconstruir la sociedad, para actuar 
sobre ella de suerte que se contribuya decidida e intencionalmente a la solución de múltiples problemas que la afectan (2006: 22).

Con esa doble voluntad en mente, el análisis hermenéutico del currículo puede pasar o incluir un segundo momento: establecer relaciones. Cuando se propone un análisis hermenéutico lo que en verdad se hace es tender puentes, anudar hilos, imbricar signos; convertir las unidades culturales en constelaciones de sentido. Este esfuerzo de ver en totalidad es el que obliga a pasar de la palabra al discurso, del elemento al código, de lo suelto a lo articulado. Cuando se establecen relaciones, se procura comprender la unidad cultural socioeducativa como parte del mundo de la vida: todo se conecta, nada opera aislado, todo apunta a un propósito. El análisis hermenéutico siempre está repleto de asociaciones que se multiplican como una red. Por eso mismo, tocar una ramificación del sentido es herir, de alguna manera, otra parte del conjunto. Se puede decir entonces, de acuerdo con lo expuesto, que los análisis sobre el currículo necesariamente tienen que abordarse, interpretarse y llevarse a la práctica desde una mirada sistémica, dado que es ahí donde es posible relacionar, conectar, ver múltiples causas y consecuencias del acto educativo. De ahí que Vasco considere en ese sentido que:

Los debates, los foros, los trabajos en grupo lo mismo que el estudio y la reflexión personal sobre los procesos en los cuales estamos inmersos, constituyen el taller donde se fragua esa versión de la Teoría General de Procesos y Sistemas que nos ayuda a comprender los procesos sociales, políticos, económicos, culturales y ambientales entre los cuales se desarrolla la educación y que no poseen límites fijos ni comportamientos rígidamente determinados, ni relaciones estáticas (1995: 113).

Ese tipo de relaciones sistémicas que intentan no dejar nada por fuera, ofrecen una perspectiva del currículo más abierta y flexible, de modo que puedan responder a cada contexto histórico.

\section{El currículo comporta una formación que sea integral}

Lo que se viene exponiendo en los apartados anteriores lleva a consolidar un currículo cuyo propósito fundamental es formar integralmente. Y en ese sentido, al calificar la formación como integral, no sólo se está subrayando el carácter formativo de la educación sino que además se está especificando el sentido de la misma, del ser de la persona. Por eso "pensamos que lo integral no es sumatoria. Que integral tiene que ver con opciones, con decisiones, con intencionalidades, con posturas y prioridades. Integral no es conjunto de, sino relación con" (Vásquez, 2008: 33). Una educación encaminada a la formación integral asume al ser humano como persona íntegra, como totalidad, es una educación que reconoce las dimensiones humanas en constante interrelación, no por partes, sino en permanente despliegue, presentes siempre en todos los ámbitos de la existencia; es una educación que reconoce que en cualquier actividad educativa está implicada la persona como un todo; en consecuencia, ni privilegia la inteligencia sobre la afectividad, ni separa la imaginación de la acción, ni hace dicotómico el desarrollo individual del social. 
Se trata de una educación que busca propiciar el desarrollo armónico de todas las dimensiones del individuo. Un desarrollo que sea armónico supone la unidad en la diversidad, porque precisamente si se retoma su sentido estético la armonía se refiere a la combinación simultánea pero diferente de varios sonidos o colores. Esto implica que un desarrollo armónico no requiere que todas las dimensiones del individuo se desarrollen de la misma manera, con la misma intensidad y al mismo tiempo. Porque ninguna entidad educativa puede darlo todo. Se trata, más bien, de propiciar el desarrollo del talento humano, de permitir que cada quien sea lo que puede ser, que avive y busque perfeccionar sus fortalezas en el contexto de la totalidad de su ser.

Precisamente, la totalidad se construye en la combinación de las diferencias; combinación que no puede reducirse a ser un mero agregado de elementos separados. "El ser humano es a la vez físico, biológico, psíquico, cultural, social e histórico. Es esta unidad compleja de la naturaleza humana la que está desintegrada en la educación y que imposibilita aprender lo que es ser humano" (Morin, 2001: 17). De ahí que más que cultivar un currículo de contenidos que promuevan la formación integral, el énfasis ha de colocarse en el proceso de constitución en unidad del ser-persona y aprender a convivir con los demás en la diferencia. En esta perspectiva, la formación integral no puede basarse en la enseñanza de competencias ni en la mera transmisión de información, sino en el aprendizaje de procedimientos que permitan seguir aprendiendo, que ofrezcan elementos para establecer conexiones entre lo que apa- rece aislado para que se asuma críticamente la cultura, de tal suerte que puedan seguirse creando nuevas maneras de ser y de actuar. Una educación enfocada a la formación integral busca superar las visiones yuxtapuestas de las diversas ciencias, culturas y técnicas haciendo énfasis en los nexos entre lo especializado y la dimensión general, buscando dar sentido a todo el proceso de la vida humana.

\section{Valor formativo desde lo racional y emocional del currículo}

Se concibe la acción curricular como la propia de una entidad educativa que la distingue de cualquier otra y se propone como el semillero de la formación integral. En esta perspectiva, primero, no se equipara al currículo con la organización de conocimientos ya que estos se asumen como una mediación, no como un fin de la educación. En esta visión el conocimiento tiene ante todo un valor formativo y no meramente productivo o explicativo. Segundo, es importante tener en cuenta que el currículo no puede entenderse como un objeto, fijo y determinado, sino más bien como un proceso y, en consecuencia, se subraya que el énfasis ha de colocarse en la acción de construir currículos, esto es, la curricularización.

Al retomar el sentido originario del término currículo, que designa el camino por recorrer, se puede entender como método que, según la etimología griega, significa camino, camino que sólo se hace mientras se recorre. Es interesante esta cercanía de los vocablos en tanto que el camino que se traza no podrá ser normativo, sino reiterativo. En consecuencia, 
el currículo no está en un recetario prescrito, tampoco puede reducirse a plan de estudios ni a la organización de conocimientos, sino que siempre es proceso formativo, posibilidad por realizarse acompañado de su razón, de su sentido, y en buena medida con una gran dosis de desarrollo de toda la vida emocional que permite garantizar gratificación y afecto en los procesos educativos. En verdad el diseño de un currículo sin educación emocional que promueva el bienestar y la formación para la ciudadanía desde la escuela se queda corto y puede reproducir los esquemas tradicionalistas de la enseñanza que se fundamentan en una educación basada en aprender conocimientos racionalmente, pero sin ningún impacto en la vida emocional y social de los grupos. Así lo ha considerado el profesor lafrancesco al afirmar:

En la institución educativa es importante para el educando, aprender con flexibilidad a establecer límites, a comprender las normas de comportamiento, a desarrollar la autodirección personal y de la conciencia, a auto-regularse y normalizarse, pues las buenas conductas y los buenos comportamientos inspiran afectividad en los demás. Es entonces, de vital importancia, que el educador-mediador sea flexible, tenga actitudes de empatía y comprensión y comprenda las necesidades de afecto de sus educandos, y ofrezca y genere estrategias para que los demás educandos y los padres de familia se comporten afectiva y empáticamente (2011: 86).

Cuando al construir el currículo se pegunta ¿qué es lo específico del sujeto humano?, ¿qué es lo que se tiene que formar en él?, la respuesta común es que es animal racional, y por lo tanto hay que formarlo para que haga buen uso de la razón. Y eso evidentemente hay que hacerlo; sin embargo, se ha magnificado este rasgo, lo cual hace que se pase por alto y se desvalorice el hecho de que, como seres biológicos, los seres humanos también son seres emocionales y que, en ese sentido, las acciones educativas necesariamente se entrelazan entre razón y emoción (y dentro de ellas todas las demás dimensiones que hacen parte de una formación integral y que ya se han venido mencionando a lo largo de este trabajo). A propósito del asunto, Echeverría considera que:

Desde el comienzo, sin embargo, hubo reacciones contrarias a esta concepción del ser humano, especialmente en cuanto al papel asignado a nuestras emociones, los así llamados "asuntos del corazón". Blaise Pascal, filósofo francés contemporáneo de Descartes, nos advirtió que el "corazón tiene sus razones que la razón desconoce". Pero a pesar de la advertencia, el cartesianismo se convirtió en nuestra forma predominante de comprender el fenómeno humano. Sostenemos que para comprender la acción humana, debemos prestar cuidadosa atención a nuestra vida emocional. Postulamos que nuestras emociones son determinantes básicas de lo que podemos o no lograr en los dominios del aprendizaje y/o sociabilidad (1996: 255-256).

Este camino no es fruto del azar ni del tanteo individual, sino que requiere de un encadenamiento razonado y con mucho afecto en pos de un proyecto que cada comunidad define. De acuerdo con esta perspectiva, se entiende la curricularización como una acción intencional, esto es, constitutiva de sentido; es lo que hace inteligible los procesos educativos. Así, curricularizar es dar sentido, pero dar sentido 
con razón y amor; dar valor formativo a cualquier actividad que se realice. Podría decirse, es donde se concreta la función orientadora de la educación en tanto que muestra caminos, trayectos que están por recorrerse.

\section{Hacia la superación de lo oculto y lo extracurricular}

Al curricularizar se toman las múltiples posibilidades de acción de la organización educativa, se las reconoce y se explícita su sentido formativo. De ahí que no pueda haber nada extra curricular en el sentido literal del término -al menos desde la mirada que trabaja este ensayo-. Lo extracurricular será lo que no es importante ni necesario para la formación. En esta perspectiva puede haber múltiples acciones que no requieren ser curricularizables, precisamente porque no se les desea atribuir valor formativo. Tampoco se puede concebir un currículo oculto, porque se trata, precisamente, de dar sentido a cualquiera de las actividades y procesos que se realicen. Si es oculto es porque no se ha visto, no se ha comprendido su posibilidad formativa. Así, por ejemplo, los tiempos libres, la biblioteca o la oración, etc., son curricularizables, no porque se integren a los procesos formalizados de educación, sino porque se les reconoce su sentido formativo por ser precisamente lo que son, y se articulan como parte de las acciones y prácticas educativas. En este sentido, se ha pronunciado Malagón de la siguiente manera:

Si la escuela tradicional estaba circunscrita a un espacio, una normatividad y unos contenidos en tiempos predeterminados, hoy el concepto de escuela involucra la vida misma, la cotidianidad, el quehacer diario y de la misma manera el currículo también es posible conceptualizarlo más allá de la predeterminación (2004: 8).

Entonces, curricularizar supone traducir -entendido el término metafóricamente-, lo cual implica convertir conocimientos y experiencias en procesos que puedan ser aprendidos por otros, en acciones con sentido que contribuyan a la formación integral, conjugar, poner en juego los diversos componentes de los procesos educativos; orientar, mostrar caminos, partiendo de lo existente para posibilitar el futuro. El currículo ha de permitir la puesta en escena de acciones con sentido, las cuales se reinterpretan para fortalecer de nuevo los procesos curriculares. En consecuencia, el énfasis al curricularizar está en el modo, en las múltiples y variadas maneras de realizar acciones educativas que desarrollen la sensibilidad, enriquezcan la imaginación y permitan la comprensión de sí mismos de quienes, como actores de estos procesos, puedan continuar creando mundos ni siquiera imaginados.

En relación con los estudiantes, la tarea de curricularizar le corresponde al profesor, que junto con toda la comunidad educativa es lo que, indistintamente de la ciencia o disciplina que enseñe, cualquier maestro debe hacer. Y esta tarea se da en varios niveles: el más cotidiano, el de la preparación de la acción de clase que al comprenderla como componente del proceso curricular deja de verse como un lugar para impartir información y se convierte en pretexto para el desarrollo de los estudiantes, de acuerdo con el sentido y el horizonte de formación integral; un nivel mediador, el de 
la elaboración de planes y programas que así entendidos dejan de ser un requisito formal para convertirse en verdaderas guías no sólo de los cursos sino de las diversas actividades de la vida estudiantil; un nivel más general, el del proyecto institucional que fundamenta y dirige las acciones de la entidad en pos de la formación integral. Y, finalmente, construye un currículo para vincularlo con la sociedad, con el entorno, para responder de manera crítica a los desafíos del mundo de hoy. En ese sentido, curricularizar pasa necesariamente por el ámbito político, social y cultural no como un "invitado de piedra", sino como parte fundamental de los procesos sociales de resistencia frente a las posibles formas de injusticia social y donde la educación cumple un papel muy importante, que aporta desde sus propias perspectivas a los procesos históricos de cambio e integración con las comunidades que buscan formarse para aportar a las dinámicas de transformación que requiere el mundo de hoy.

Los debates esenciales en torno a los currículos en la actualidad, como no podía ser de otro modo, están muy estrechamente relacionados con los cambios culturales, políticos, sociales y económicos que están afectando a las sociedades desarrolladas y que tienen como primera consecuencia la revisión del papel asignado a la escolarización y a las relaciones entre ésta y los diferentes aspectos que en ella se entrecruzan: profesores, organizaciones, relaciones con la comunidad (Sacristán, 1997: 17).

Y si curricularizar es la principal tarea en la que se centra la formación integral, esta acción no puede ser sólo una forma de organizar los conocimientos, sino que debe permear toda la vida de la institución educativa, precisamente para que sea entidad educativa en todo el sentido de la palabra. En consecuencia, el trabajo de apoyo social, o actividades de tipo cultural, social, deportivo o religioso no pueden ir paralelas al trabajo académico, ellas son también académicas por ser formativas. Asumir esta perspectiva implica que cualquiera de las actividades hasta ahora consideradas extracurriculares haga parte de manera explícita de los procesos académicos de los estudiantes. Esto no significa que deban perder su particularidad para ajustarse a los parámetros que aún rigen algunos procesos formalizados. Se trata de incorporar prácticas lúdicas, estéticas, gregarias y de hondo sentido espiritual no como una adición, sino como parte intrínseca de los procesos educativos.

Estos mismos aspectos también deben intervenir en toda la vida laboral de la entidad educativa para que el trabajo también se constituya en posibilitador de la formación integral. De esta manera, la administración estará no sólo en función de la academia, sino beneficiándose de ella, porque las formas de trabajo también reflejarán la intencionalidad de la formación integral que atraviesa el currículo. El trabajo administrativo cobra valor en relación con las acciones formativas que desde ahí se generan tanto con los estudiantes como con todo el personal. En cierta manera, el término administración sufre un desplazamiento por el de animación, que se concibe como "una práctica que pretende reafirmar la vida en la colectividad" (Velásquez, 1997: 39). 
Los profesores en su mismo desempeño encuentran estímulos para reconocer que nunca les basta lo que aprendieron de estudiantes, que no pueden ellos prescindir de estar repasando, rectificando ideas envejecidas, enterándose de nuevo, conociendo lo que hagan los colegas, entregando sus propias innovaciones al comentario de ellos. Si este estilo formativo se irradia a toda la entidad educativa, se estará curricularizando como comunidad académica -no como industriales, ni comerciantes, ni financistas...-, se estará construyendo un tipo especial de comunidad, una cultura propia del ser educativo que de uno $u$ otro modo busca contribuir al desarrollo integral de quienes en ella participan, y esto habrá de irradiar en las familias, en los barrios, en las ciudades, en ir inventando un nuevo tipo de país, el cual depende mucho también del tipo de sistema educativo que se implemente.

\section{Conclusiones}

De esta manera, cómo se construya el currículo depende de una formación que responda a los desafíos de la educación para el siglo XXI. Pero "esa forma" de concebir el currículo no ha de "casarse" con ninguna teoría en especial, dado que todas, de una u otra manera, aportan al diseño de prácticas educativas importantes. Lo que sí se debe tener en cuenta son las características que debe comportar una concepción práctica del currículo en los sistemas educativos que favorezcan indudablemente el desarrollo de las personas y abran a las instituciones escolares grandes posibilidades para garantizar una formación en verdad integral. Algunas de las características que se describen a continuación aparecen en las propuestas de autores como Stenhouse (1994) Sacristán (1991), Forero (1993), Kemmis, (1998), Lundgreen (1998), López (1998), Giroux (2006), Magendzo (2006), Portela (2003), Malagón (2004), lanfrancesco (2011), entre otros.

- Debe responder a las demandas históricas emergentes en lo social, político, económico y cultural.

- Debe ser pertinente de acuerdo con cada contexto histórico-cultural particular.

- Generar procesos de transformación en las comunidades educativas.

- Debe ser flexible y evitar todo intento por unificar y predeterminar los procesos de formación.

- Ha de cultivar la interdisciplinariedad como factor importante para enriquecer no sólo los conocimientos, sino también las prácticas educativas.

- Debe ser integral y sistémico para enfrentar diversos problemas multicausales que requieran diversas respuestas.

- Debe ser crítico y liberador-emancipador de todas aquellas estructuras educativas que intentan reproducir los sistemas políticos de injusticia social y exclusión.

- Formar en valores de acuerdo con aquellos a los que cada cultura cree que son importantes (ha acordado) para el desarrollo humano. 
- Fomentar la educación emocional como factor clave de éxito en procesos de transformación escolar.

- Formar en contenidos y prácticas espirituales, de acuerdo con la cultura.

- Construir una mirada curricular para los desafíos de la globalización y los grandes problemas ecológicos que enfrenta el planeta.

- Cultivar la convivencia pacífica y la resolución de conflictos desde la escuela al promover contenidos, prácticas y ambientes de aprendizaje.

\section{Referencias}

Echeverría, R. (1996). Ontología del lenguaje. Santiago de Chile: Dolmen.

Giroux, H. (2006). La escuela y la lucha por la ciudadanía. Madrid: Siglo XXI Editores.

Habermas, J. (2008). Conciencia moral y acción comunicativa. Madrid: Editorial Trotta.

lafrancesco, G. (2011). Modelo pedagógico holístico transformador. Fundamentos, dimensiones, programas y proyectos en la escuela transformadora. Bogotá: CORIPET.

Magendzo, A. (2004). Formación ciudadana. Cultura democrática. Bogotá: Transversales Magisterio.
Magendzo, A. (2006). Currículum, educación para la democracia en la modernidad. Santiago de Chile: PIIE.

Malagón, L. (2004). El currículo: dispositivo pedagógico para la vinculación universidadsociedad. Revista Electrónica de la Red de Investigación Educativa, 1(1).

Morin, E. (2001). Los siete saberes necesarios para la educación del futuro. Bogotá: ICFES.

Noguera, C. (1997). Educación y democracia: un problema más allá de la escuela y el maestro. Revista Educación y Ciudadanía, 3. Bogotá: IDEP.

Portela, H. (2003). La dialéctica de lo curricular y lo formativo... Una relación indisoluble. En Simposio sobre currículo. Bogotá: Ideaf.

Quintero, M., Ruiz, A., et ál. (2004). ¿Qué significa educar en valores hoy? Barcelona: Octaedro Oei.

Sacristán, J. (1997). Políticas y prácticas culturales en las escuelas. Los abismos de la etapa posmoderna. Buenos Aires: Editorial San Luis.

Sopó, Á. (2002). Lectura pedagógica de la hermenéutica del profesor Gadamer. Revista Itinerario Educativo, 39-40. 
Touraine, A. (2000). Crítica de la modernidad. Bogotá: Fondo de Cultura Económica.

Vasco, C. (1995). La teoría general de procesos y sistemas. En Documento de la misión ciencia, educación y desarrollo. Bogotá.
Vásquez, F. (2008). Educar con maestría. Bogotá: CMYK Ediciones. 